\title{
Postexercise heart rates and pulse palpation as a means of determining exercising intensity in an aerobic dance class
}

Department of Physiology and Pharmacology, Medical School, Queen's Medical Centre,

Nottingham NG7 2UH, United Kingdom

J M Bell, research assistant

E J Bassey, senior lecturer

Correspondence to:

Dr E J Bassey.

Accepted for publication

4 July 1995

J M Bell, E J Bassey

\begin{abstract}
Objective-To establish the accuracy of the traditional method of measuring the intensity of exercise in aerobic dance classes, that is, intermittent pulse palpation performed during a brief cessation of activity.

Methods-A short wave telemetry system was used to record heart rates during a class in a group of 12 healthy women aged 26 (SD 6) years. Subjects palpated their pulses for $10 \mathrm{~s}$ following high and low intensity exercise $[78(8) \%$ and $69(9) \%$ of mean predicted maximum heart rate respectively]. Recorded exercising heart rates, averaged over $60 \mathrm{~s}$ preceding pulse palpation [ExHR(rec)], were compared with the recorded postexercise heart rates averaged over the $10 \mathrm{~s}$ palpation period [PostExHR(rec)] and with the palpated counts (PalpHR). Differences were assessed using Student's $t$ test and Wilcoxon signed rank test.
\end{abstract}

Results-Differences between ExHR(rec) and PostExHR(rec) following high and low intensity exercise $\left[3(6)\right.$ beats $\cdot \mathrm{min}^{-1}$ and $5(7)$ beats $\cdot \mathrm{min}^{-1}$ respectively] were not significant. However, the wide variation between subjects means that a postexercise heart rate is unreliable as a measure of individual exercise intensity. PalpHR was significantly lower than ExHR(rec) $(P<0.01)$. Every individually palpated count underestimated the exercising heart rate (range 9 to 95 beats $\cdot \mathrm{min}^{-1}$ ).

Conclusions-While postexercise heart rate adequately represents the exercise heart rate for a group, the individual variation is too wide for this to be a useful measurement.

(Br F Sports Med 1996;30:48-52)

Key terms: aerobic dance; heart rates; pulse palpation

Aerobic dance consists of sequences of choreographed movements performed rhythmically to music. It has been shown to raise heart rates to levels above $55 \%$ of maximum heart rate range, and therefore to constitute a viable cardiovascular training mode. ${ }^{1}{ }^{2}$ However, unlike training modes such as running, in which intensity may be controlled by adjusting speed or gradient, aerobic dance is a free moving activity in which energy expenditure is highly variable and not amenable to precise prescription. Consequently in most community based exercise classes postexercise pulse monitoring is used to determine whether the intensity of the activity falls within a heart rate range which is safe but above the threshold required to stimulate a training effect. Despite the popularity of this practice there has been little evaluation of the efficacy of the technique. Pollock et $a l^{3}$ have published data suggesting that a postexercise palpated pulse count can adequately represent the exercising heart rate, but other investigators have questioned its validity. ${ }^{45}$

Palpating the pulse during dance is not possible and so participants monitor their pulses during a brief cessation in activity. The assumptions underlying the theory that this postexercise heart rate provides a reliable measure of the heart rate obtaining during the preceding period of exercise are twofold: firstly, that the rate at which the heart rate decreases does not vary greatly between individuals or with the intensity of the preceding activity; secondly, that the pulse can be counted accurately when its location and the initiation of the count is rapid, and the counting period is abbreviated to 6 or 10 seconds. The objectives of this study were therefore to obtain descriptive information about heart rate profiles during aerobic dance and to evaluate whether heart rate determined by palpation and after activity has ceased is a reliable method of determining exercising heart rate.

\section{Methods}

Subjects were 12 female class participants aged 26 (SD 5.6) years and with a body mass of $56 \cdot 5(6 \cdot 0) \mathrm{kg}$. All subjects had been attending the selected class during the four preceding weeks and had been advised to exercise within a recommended heart rate range which represented $60-80 \%$ of age adjusted predicted maximum in healthy women, adapted from Cotes $^{6}$ [220-0.65 age (years)]. The 15 min cardiovascular component of the class was preceded by a $10 \mathrm{~min}$ warm up. The intensity of the dance sequences which comprised the cardiovascular section was gradually increased during the first $3 \mathrm{~min}$, was maintained for $9 \mathrm{~min}$ at the increased intensity level, and was then gradually reduced during the final $3 \mathrm{~min}$. In line with current United Kingdom certification standards (RSA/Sports Council Certificate in the Teaching of Exercise to Music), participants were instructed in ways in which they could alter the intensity of the activity by, for instance, increasing or decreasing the range of movement or adapting 
high impact movements such as jogging so that one foot would remain in contact with the floor. Intermittent pulse monitoring was part of the usual format of the class and all subjects had been trained in the procedure and were aware of its purpose.

The investigation was explained and informed consent was obtained from each subject. Heart rates were recorded throughout the class using the "Sport Tester PE3000" short wave telemetry system (Polar Electro) which has been validated against conventional ECG recording. ${ }^{7}$ The receiver, which is usually worn as a wrist watch, was programmed to display and store heart rates averaged over $5 \mathrm{~s}$ intervals. It was then attached to the subjects' clothing in such a way that the heart rate display was not visible to the wearer. Heart rate recording was begun individually by the observer and the start time of each receiver relative to a master watch was noted. Starting the recording on an individual basis was considered more secure than attempting to start all watches at the same time which, in the event that any one receiver had failed to start recording at the precise moment, would have entailed resetting every other receiver before recording could be reinitiated and the class could begin.

During the $45 \mathrm{~min}$ class the participants were told on two separate occasions to stop exercising and to monitor their pulse rates over a period of $10 \mathrm{~s}$; the first occasion followed a period of high intensity dance and the second was toward the end of the aerobic component following a period of less intense activity. The master watch was used to note, on both occasions, the exact times when the class was instructed first to "stop exercising and locate your pulse", second to "start counting NOW", and finally to "stop counting NOW". On termination of the pulse count each subject's verbally reported palpated count was noted by an observer.

After the class the data stored in the receiver memory were transferred, using an interface unit, to a BBC series B computer and a display of the heart rate changes with time and a listing of heart rate values was obtained for each subject.

\section{ANALYSIS}

The starting time of each subject's recorded data relative to the master watch was used to identify on the individual listings the $5 \mathrm{~s}$ interval (following higher and lower intensity exercise) in which exercise ceased and the $5 \mathrm{~s}$ interval in which the $10 \mathrm{~s}$ palpated count was initiated. Figure 1 illustrates the identification of these time intervals. Comparisons were then made between the mean exercising heart rate (beats $\cdot \mathrm{min}^{-1}$ ) recorded over $60 \mathrm{~s}$ before cessation of exercise [ExHR(rec)] and the mean heart rate recorded during the $10 \mathrm{~s}$ palpation period [PostExHR(rec)]. This determined the accuracy with which the heart rate recorded during pulse palpation approximated the heart rate recorded during the preceding exercise. In addition both ExHR(rec) and PostExHR(rec) were compared with the verbally reported palpated rates (PalpHR). This determined the accuracy with which the palpated counts approximated the heart rates recorded during both exercise and pulse palpation. Heart rate
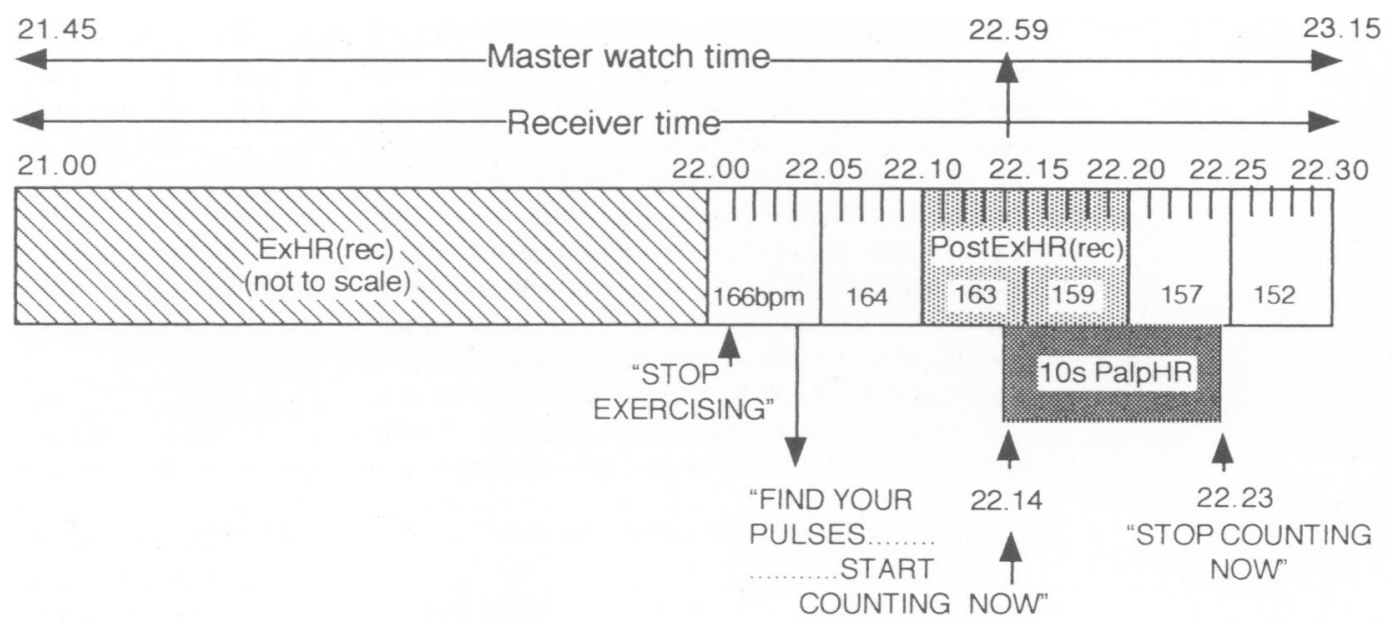

Exercising Heart Rate [ExHR(rec)] was determined by averaging the twelve $5 \mathrm{~s}$ bands which preceded the band in which the class was told to "STOP EXERCISING"

Post-exercise Heart Rate [PostExHR(rec)] was determined by averaging the HRs of the $5 s$ band in which the palpated count commenced and the subsequent band

The moment that the palpated count commenced was identified on each print-out by subtracting the difference between the master watch and individual start times (e.g. for this subject $45 \mathrm{~s}$ ) from 22.59 which was the time on the master watch when the instructor said "NOW" (the signal to start counting)

The example illustrates the maximum possible mismatch between 10 s palpated count and $10 \mathrm{~s}$ PostEx(rec)

Fig 1 An illustration of one subject's recorded data to show the identification of various time bands from which heart rate $(H R)$ data were taken for analysis. 
means over the entire $15 \mathrm{~min}$ workout were also obtained to determine the percentage of the individual's age adjusted maximum achieved.

Given the $5 \mathrm{~s}$ mean value recorded by the telemetry system and the individual start time of each subject's recorded data, there was, for some subjects, an inevitable mismatch between the $10 \mathrm{~s}$ postexercise periods used to compare recorded and palpated counts. Examination of the raw data revealed that this was the case in 15 out of 21 possible instances. To calculate the maximum potential discrepancy between palpated and recorded counts which might have arisen from the mismatches, the postexercise heart rate was in each case recalculated using two alternative approaches: first, the mean of the three $5 \mathrm{~s}$ intervals which the mismatched palpated count spanned was calculated; and second, the $5 \mathrm{~s}$ band in which the palpated count was initiated was ignored and the mean of the subsequent two $5 \mathrm{~s}$ intervals was calculated. These "modified" recorded rates were then compared with those established using the original method [PostExHR(rec)]. The maximum difference in values was \pm 3 beats $\cdot \mathrm{min}^{-1}$. Figure 1 replicates one subject's recorded data in which there was a maximal potential discrepancy of +3 beats $\cdot \mathrm{min}^{-1}$.

Although the data were not skewed they were kurtosed, and differences assessed using Student's $t$ test for paired means were therefore also assessed using Wilcoxon signed rank test. The 0.05 level of significance was selected.

\section{Results}

Descriptive data are given as means (SD) unless otherwise stated.

The mean heart rate achieved over the 15 min workout, expressed as a percentage of individual age adjusted heart rate maximum, was $70 \cdot 9(5 \cdot 8) \%$. The mean exercising heart rate for the group during higher and lower intensity exercise was $78 \cdot 5(8 \cdot 2) \%$ and $68 \cdot 5(8 \cdot 8) \%$ of predicted maximum respectively (table). There was, however, wide individual variation within both higher and lower intensity exercise, with rates ranging from $65-92 \%$ and $54-85 \%$ of predicted maximum respectively. Three subjects during higher intensity exercise and two during lower intensity work exceeded the upper limit of their recommended range.

There was no significant difference for the group between ExHR(rec) and PostExHR(rec) (fig 2) following higher or lower intensity work. The absolute decrease in rate following lower intensity exercise $\left[5(7)\right.$ beats $\left.\cdot \mathrm{min}^{-1}\right]$ was not significantly different from the decrease in rate following higher intensity exercise [3(6) beats $\cdot \mathrm{min}^{-1}$ ]. There was, however, considerable individual variation which ranged from a 15 beat $\cdot \mathrm{min}^{-1}$ decrease to a $3 \mathrm{beat} \cdot \mathrm{min}^{-1}$ increase following high intensity exercising, and from a 15 beat $\cdot \mathrm{min}^{-1}$ decrease to a 9 beat $\cdot \min ^{-1}$ increase following lower intensity exercise. Individual variation was less evident following high intensity work but following low intensity work PostExHR(rec) diverged from $\operatorname{ExHR}(\mathrm{rec})$ by more than $5 \%$ in seven of the 12 subjects.

One subject was unable to locate and palpate her pulse on either occasion. All remaining subjects reported palpated counts which underestimated the exercising heart rate with discrepancies which ranged from 9 to 95 beats $\cdot \min ^{-1}$. Following high and low intensity work PalpHR underestimated $\operatorname{ExHR}(\mathrm{rec})$ by $29(26)$ beats $\min ^{-1}[18 \cdot 4(15 \cdot 3) \%]$ and $22(15)$ beats $\min ^{-1}[16 \cdot 2(12 \cdot 2) \%]$, respectively. Both

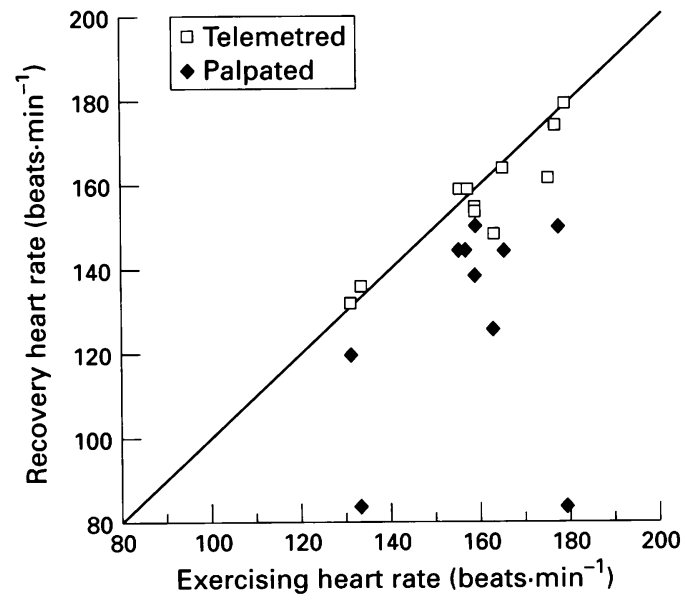

Fig 2 Individual postexercise heart rates (palpated and recorded) plotted against heart rates during high intensity exercise. The line of identity is drawn. (Subject 7 is excluded because of faulty telemetry.)

Physical characteristics and mean recorded exercising heart rates (beats $\cdot \mathrm{min}^{-1}$ ) during higher and lower intensity dance for 12 female subjects

\begin{tabular}{|c|c|c|c|c|c|c|}
\hline \multirow{2}{*}{$\begin{array}{l}\text { Subject } \\
\text { No }\end{array}$} & \multirow{2}{*}{$\begin{array}{l}\text { Age } \\
\text { (years) }\end{array}$} & \multirow{2}{*}{$\begin{array}{l}\text { Weight } \\
(\mathrm{kg})\end{array}$} & \multicolumn{2}{|l|}{ Higher intensity } & \multicolumn{2}{|l|}{ Lower intensity } \\
\hline & & & $\begin{array}{l}\text { Exercising } H R \\
{[E x H R(\text { rec })]}\end{array}$ & $\begin{array}{l}\% A g e^{\mathrm{a}} \text { adjusted } \\
\text { maximum }\end{array}$ & $\begin{array}{l}\text { Exercising } H R \\
{[E x H R(r e c)]}\end{array}$ & $\begin{array}{l}\% \text { Age adjusted } \\
\text { maximum }\end{array}$ \\
\hline $\begin{array}{r}1 \\
2 \\
3 \\
4 \\
5 \\
6 \\
7 \\
8 \\
9 \\
10 \\
11 \\
12\end{array}$ & $\begin{array}{l}25 \\
26 \\
32 \\
40 \\
28 \\
19 \\
21 \\
25 \\
28 \\
21 \\
23 \\
25\end{array}$ & $\begin{array}{l}51 \cdot 8 \\
53 \cdot 6 \\
58 \cdot 6 \\
56 \cdot 4 \\
50 \cdot 1 \\
70 \cdot 0 \\
60 \cdot 5 \\
56 \cdot 4 \\
51 \cdot 8 \\
61 \cdot 4 \\
58 \cdot 7 \\
48 \cdot 2\end{array}$ & $\begin{array}{l}156 \\
131 \\
133 \\
179 \\
157 \\
165 \\
t \\
159 \\
177 \\
159 \\
163 \\
175\end{array}$ & $\begin{array}{l}76 \\
65 \\
66 \\
92 \\
77 \\
79 \\
\dagger \\
78 \\
88 \\
77 \\
79 \\
86\end{array}$ & $\begin{array}{l}135 \\
110 \\
117 \\
166 \\
131 \\
136 \\
168 \\
144 \\
148 \\
136 \\
143 \\
136\end{array}$ & $\begin{array}{l}66 \\
54 \\
58 \\
85 \\
64 \\
65 \\
82 \\
71 \\
73 \\
67 \\
70 \\
67\end{array}$ \\
\hline $\begin{array}{l}\text { Mean } \\
\text { SD }\end{array}$ & $\begin{array}{r}26 \cdot 0 \\
5 \cdot 6\end{array}$ & $\begin{array}{r}56 \cdot 5 \\
6 \cdot 0\end{array}$ & $\begin{array}{c}160 \dagger \\
16\end{array}$ & $\begin{array}{c}78 \cdot 5 \dagger \\
8 \cdot 2\end{array}$ & $\begin{array}{r}139 \\
17\end{array}$ & $\begin{array}{r}68 \cdot 5 \\
8 \cdot 8\end{array}$ \\
\hline
\end{tabular}

HR, heart rate.

a Age adjusted predicted maximum $\mathrm{HR}=220-(0.65 \times \mathrm{age})$ beats $\cdot \mathrm{min}^{-1}$

tSubject 7's recorded heart rate was faulty during higher intensity exercise and has therefore been excluded. 
differences were significant $(P<0.01)$. In all but one case PalpHR also underestimated the PostExHR(rec). The differences following high and low intensity work were 27(27) and $16(18)$ beats $\mathrm{min}^{-1}$, respectively. These differences were significant at the higher intensity level $(P<0.01)$ and at the lower intensity level (Student's $t$ test $\mathrm{P}<0.05$, Wilcoxon signed rank test $P<0.01$ ). In only three instances were the palpated underestimations sufficiently modest that they could be accounted for by the potential discrepancy arising from the mismatch between recorded and palpated time periods which, as previously described, was calculated to be at maximum \pm 3 beats $\cdot \mathrm{min}^{-1}$.

\section{Discussion}

For all subjects, mean exercising heart rates for the 15 minute programme exceeded the recommended training threshold of $55 \%$ maximum heart rate (ACSM 1991) which shows that aerobic dance taught in a community setting is a viable cardiovascular training mode. There was, however, a wide variation in heart rate response, with some participants exceeding the recommended upper limit of $80 \%$ age predicted maximum during both higher and lower intensity exercise. This is of concern for community based classes without medical back up and highlights the need for an effective method of monitoring the intensity of the activity.

The findings of the present investigation show that for the group a postexercise heart rate measurement adequately represents the exercising heart rate. Differences of less than 5 beats $\cdot \mathrm{min}^{-1}$, especially if consistent following work of varying intensity, would mean that for most practical purposes a postexercise rate would represent a useful measure of the intensity of the preceding activity. The mean values, however, conceal wide individual variation which makes the practice unacceptable for individual monitoring and even potentially dangerous in the case of the elderly or unfit.

Evidence for a systematic relation between exercising and postexercise heart rates is confined to studies which have controlled the relative intensity of the preceding exercise. Linnarsson ${ }^{8}$ has shown that the rate of change in heart rate during recovery becomes slower with both increasing absolute and relative workloads, the relative stress of the exercising workload being the crucial factor in the heart rate response at the end of exercise. Similarly Pollock et $a l^{3}$ have reported that when exercising intensity is at or in excess of $80 \%$ of individual maximum, recovery rate within the first 12-14 seconds will vary from the exercising rate by less than $2 \%$.

The free moving nature of dance means that absolute and therefore relative intensity levels cannot be prescribed, besides which the functional capacity of those attending community classes cannot be determined. Factors such as genetic endowment, age, gender, habitual activity levels, and motor skills will all contribute to differences in the relative intensity which the movements represent for individuals. In addition the practice of encouraging participants to keep their feet moving, to aid venous return on cessation of exercise, will affect the rate of heart rate deceleration to differing degrees depending on the vigour with which this is performed. In the present study the 9 beat $\cdot \mathrm{min}^{-1}$ increase in recorded postexercise heart rate observed in one subject was almost certainly attributable to the subject having stood still to locate her pulse.

Clearly the biggest source of error in a postexercise pulse count is the variation in individual ability to palpate the pulse accurately. Doust $^{5}$ has suggested that, after cessation of exercise, concentration and effort are required to coordinate the location and counting of the pulse. In addition, surrounding noise, increased respiration rate, and the need to keep the feet moving to avoid venous pooling will all exacerbate an intrinsically difficult task. Moreover at higher heart rates the task of identifying distinct heart beats becomes more difficult; for example at a rate of 180 beats $\cdot \mathrm{min}^{-1} 3$ beats $\cdot \mathrm{s}^{-1}$ have to be distinguished. An abbreviated counting period inevitably compounds any such errors; a discrepancy of one beat over 10 seconds will, for instance, result in an error of 6 beats $\cdot \mathrm{min}^{-1}$.

In this study, despite familiarising the subjects with both the procedure and its rationale, one subject failed to locate her pulse on either occasion and nine reported pulse counts of either $20\left(120\right.$ beats $\left.\cdot \mathrm{min}^{-1}\right)$ or 25 ( 150 beats $\cdot \mathrm{min}^{-1}$ ) which may indicate a tendency to report a "round" number if asked. Such inaccuracy is in contrast to the findings of Williams and Morton, ${ }^{9}$ who reported a close correlation between self monitored and recorded heart rates across intensities which ranged from rest to $85 \%$ of maximum heart rate. Individual discrepancies were, however, not reported.

Traditionally aerobic dance studies have relied on pulse palpation as the only method of monitoring the intensity of the training programme $\mathrm{e}^{10-13}$ but in the light of the present investigation training outcomes should be interpreted with caution. Koltyn and Morgan ${ }^{14}$ have shown that ratings of perceived exertion (RPE) scales described by Borg ${ }^{15}$ are more effective than pulse palpation as a means of monitoring intensity during aerobic dance training. However, Grant et al ${ }^{16}$ reported that during an exercise session run on similar lines to aerobic dance, RPE values continued to rise during the class despite the percentage of maximum oxygen uptake and heart rate reserve remaining relatively constant and well matched. Further investigation, both under laboratory conditions and in community settings, needs to be undertaken to establish whether RPE does constitute a reliable means of monitoring intensity during aerobic dance. In the meantime, especially within community classes, encouraging participants to monitor symptoms such as breathlessness and fatigue on a continuous basis may be the most appropriate means of ensuring that participants exercise at a level which is safe but great enough to elicit a training effect. Symptoms which are perceived as creating discomfort rather than an 
acceptable sense of exertion would be the criterion for participants to reduce either the absolute intensity of the activity or the relative contribution from upper or lower body musculature. In practice, modification of workload in response to perceived exertion levels probably occurs spontaneously during free moving activities ${ }^{217-19}$ and may be the reason why aerobic dance classes, despite their popularity, have a low incidence of cardiac or other serious events.

We are grateful to Mrs Helen Tomlinson (née Wright) who instructed the aerobic dance class.

1 ACSM. Guidelines for exercise testing and prescription, 4th ed. Lea \& Febiger, 1991

2 Bell J M, Bassey E J. A comparison of the relation between oxygen uptake and heart rate during different styles of aerobic dance and a traditional step test in women. Eur f Appl Physiol 1994;68:20-4.

3 Pollock M L, Broida J, Hendrick Z. Validity of the palpation technique of heart rate determination and its estimation of training heart rate. Res $Q 1972 ; 43: 77-81$.

4 McArdle $\mathrm{W} D$, Zwiren L, Mager J R. Validity of post exercise heart rate as a means of estimating heart rate during work of varying intensities. Res $Q 1969 ; 40: 523-8$.

5 Doust J H. Self-paced exercise: a study of the underlying physiological and perceptual responses. Nottingham: University of Nottingham, 1983 [PhD thesis].

6 Cotes J E. Lung function, 3rd ed. Oxford: Blackwell, 1975. stability and functionality. Physician Sports Med 1988;16: stability
8 Linarsson D. Dynamics of pulmonary gas exchange and heart rate changes at start and end of exercise. Acta Physiol heart rate changes at start

9 Williams L D, Morton A R. Changes in selected cardiorespiratory responses to exercise and in body composition following a 12 week aerobic dance programme. $\mathcal{f}$ Sport Sci $1986 ; 4: 189-99$.

10 Dowdy D B, Cureton K J, Duval H P, Ouzts H G. Effect of aerobic dance on physical work capacity, cardiovascular function and body composition of middle-aged women. Res $Q$ 1985;56:227-33.

11 Williford $\mathrm{H} \mathrm{N}$, Blessing D L, Barksdale J M, Smith F H. The effects of aerobic dance training in serum lipids, lipoproteins and cardiopulmonary function. 7 Sports $M e d$ Phys Fitness 1988;28:151-7.

12 Perry A, Mosher P, La Perriere A, Roalstad M, Ostrovsky P. A comparison of training responses to interval versus continuous aerobic dance. $\mathcal{f}$ Sports $\mathrm{Med}$ Phys Fitness 1988;28:274-9.

13 Hopkins D R, Murral B, Hoeger W W, Rhodes C. Effect of low impact aerobic dance on the functional fitness of olderly women. Gerontologist 1990;30:189-92.

14 Koltyn K F, Morgan W P. Efficacy of perceptual versus heart rate monitoring in the development of endurance. Br F Sports Med 1992;26:132-4.

15 Borg G. Psychophysical bases of perceived exertion. Med Sci Sports Exerc 1982;14:377-87.

16 Grant S, Armstrong G, Sutherland R, Wilson J Aitchisont T, Paul E, et al. Physiological and psychological responses to a university fitness session. $\mathrm{Br} F$ Sports Med 1993;27:162-7.

17 Blessing D L, Wilson G D, Puckett J R, Ford H T. The physiological effects of eight weeks of aerobic dance with and without hand-held weights. Am f Sports Med 1987; 15:508-10.

18 Thomsen I A, Ballor D. Physiological responses during aerobic dance of individuals grouped by aerobic capacity and dance experience. Res $Q 1991 ; 62: 68-72$.

19 Smith J, Bishop P, May E. Addition of handheld weights to aerobic dance exercise does not alter aerobic fitness changes [abstr]. Med Sci Sports Exerc 1989;19:S89. 